Article

\title{
New Results on Qualitative Behavior of Second Order Nonlinear Neutral Impulsive Differential Systems with Canonical and Non-Canonical Conditions
}

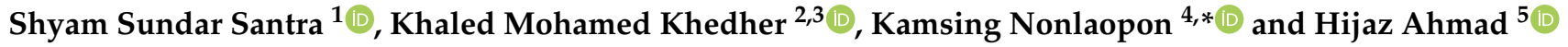 \\ 1 Department of Mathematics, JIS College of Engineering, Kalyani, West Bengal 741235, India; \\ shyam01.math@gmail.com or shyamsundar.santra@jiscollege.ac.in \\ 2 Department of Civil Engineering, College of Engineering, King Khalid University, Abha 61421, Saudi Arabia; \\ kkhedher@kku.edu.sa \\ 3 Department of Civil Engineering, High Institute of Technological Studies, Mrezgua University Campus, \\ Nabeul 8000, Tunisia \\ 4 Department of Mathematics, Faculty of Science, Khon Kaen University, Khon Kaen 40002, Thailand \\ 5 Department of Basic Sciences, University of Engineering and Technology, Peshawar 25000, Pakistan; \\ f17ppbsi011@uetpeshawar.edu.pk or hijaz555@gmail.com \\ * Correspondence: nkamsi@kku.ac.th
}

check for updates

Citation: Santra, S.S.; Khedher, K.M.; Nonlaopon, K.; Ahmad, H. New Results on Qualitative Behavior of Second Order Nonlinear Neutral Impulsive Differential Systems with Canonical and Non-Canonical Conditions. Symmetry 2021, 13, 934. https://doi.org/10.3390/sym13060934

Academic Editors: Aviv Gibali and Calogero Vetro

Received: 24 April 2021

Accepted: 20 May 2021

Published: 25 May 2021

Publisher's Note: MDPI stays neutral with regard to jurisdictional claims in published maps and institutional affiliations.

Copyright: (C) 2021 by the authors Licensee MDPI, Basel, Switzerland. This article is an open access article distributed under the terms and conditions of the Creative Commons Attribution (CC BY) license (https:// creativecommons.org/licenses/by/ $4.0 /)$.
Abstract: The oscillation of impulsive differential equations plays an important role in many applications in physics, biology and engineering. The symmetry helps to deciding the right way to study oscillatory behavior of solutions of impulsive differential equations. In this work, several sufficient conditions are established for oscillatory or asymptotic behavior of second-order neutral impulsive differential systems for various ranges of the bounded neutral coefficient under the canonical and non-canonical conditions. Here, one can see that if the differential equations is oscillatory (or converges to zero asymptotically), then the discrete equation of similar type do not disturb the oscillatory or asymptotic behavior of the impulsive system, when impulse satisfies the discrete equation. Further, some illustrative examples showing applicability of the new results are included.

Keywords: oscillation; non-oscillation; nonlinear; delay argument; canonical; impulse; non-canonical

\section{Introduction}

As is well known, impulsive differential equations serve as basic models to study the dynamics of processes that are subject to sudden changes in their states. We see symmetry every day but often do not realize it. People use concepts of symmetry, including translations, rotations, reflections, modelling and tessellations as part of their careers. In this paper, we use the concept of symmetry slightly to study the oscillation criteria of neutral impulsive differential equations. Next, we are highlighting some current developments of oscillation theory to impulsive differential systems.

In [1], Shen and Wang studied the impulsive systems of the form

$$
\left\{\begin{array}{l}
v^{\prime}(\lambda)+q(\lambda) v(\lambda-\varsigma)=0, \quad \lambda \neq \varphi_{\iota}, \quad \lambda \geq \lambda_{0} \\
v\left(\varphi_{\iota}^{+}\right)-v\left(\varphi_{\iota}^{-}\right)=I_{l}\left(v\left(\varphi_{\iota}\right)\right), \quad \iota \in \mathbb{N}
\end{array}\right.
$$

where $q, I_{\iota} \in C(\mathbb{R}, \mathbb{R})$ for $\iota \in \mathbb{N}$, and obtained new oscillation results for (1).

In [2], Graef et al. have studied the impulsive system

$$
\left\{\begin{array}{l}
(v(\lambda)-p(\lambda) v(\lambda-\delta))^{\prime}+q(\lambda)|v(\lambda-\varsigma)|^{\mu} \operatorname{sgn} v(\lambda-\varsigma)=0, \quad \lambda \geq \lambda_{0} \\
v\left(\varphi_{\iota}^{+}\right)=I_{\iota} v\left(\varphi_{\iota}\right), \quad \iota \in \mathbb{N}
\end{array}\right.
$$

assuming that $p(\lambda) \in P C\left(\left[\lambda_{0}, \infty\right), \mathbb{R}_{+}\right)$(that is, $p(\lambda)$ is piecewise continuous in $\left.\left[\lambda_{0}, \infty\right)\right)$, studied the oscillation criteria of (2). 
In [3], the authors established some new oscillation criteria for first order impulsive neutral delay differential systems of the form

$$
\left\{\begin{array}{l}
(v(\lambda)-p(\lambda) v(\lambda-\delta))^{\prime}+q(\lambda) v\left(\lambda-\varsigma_{1}\right)-q_{2}(\lambda) v\left(\lambda-\varsigma_{2}\right)=0, \quad \varsigma \geq \delta>0 \\
v\left(\varphi_{\iota}^{+}\right)=I_{\iota}\left(v\left(\varphi_{\iota}\right)\right), \quad \iota \in \mathbb{N}
\end{array}\right.
$$

under the assumptions that $p(\lambda) \in P C\left(\left[\lambda_{0}, \infty\right), \mathbb{R}_{+}\right)$and $b_{\iota} \leq \frac{I_{l}(v)}{v} \leq 1$.

Oscillation and non-oscillation properties of second-order impulsive system were studied by Tripathy and Santra in [4], where the authors considered the problem

$$
\left\{\begin{array}{l}
(v(\lambda)-p v(\lambda-\delta))^{\prime \prime}+q_{1} v(\lambda-\varsigma)=0, \quad \lambda \neq \varphi_{\iota}, \quad \iota \in \mathbb{N} \\
\Delta\left(v\left(\varphi_{\iota}\right)-\tilde{p} v\left(\varphi_{\iota}-\delta\right)\right)^{\prime}+q_{2} v\left(\varphi_{\iota}-\varsigma\right)=0, \quad \iota \in \mathbb{N},
\end{array}\right.
$$

where all coefficients and delays are constant. Other sufficient and necessary conditions for qualitative properties of second-order impulsive system were obtained in [5], where Tripathy and Santra studied the impulsive system of the form

$$
\left\{\begin{array}{l}
\left(r(\lambda)(v(\lambda)+p(\lambda) v(\lambda-\delta))^{\prime}\right)^{\prime}+q_{1}(\lambda) g(v(\lambda-\varsigma))=0, \quad \lambda \neq \varphi_{\iota}, \quad \iota \in \mathbb{N} \\
\Delta\left(r\left(\varphi_{\iota}\right)\left(v\left(\varphi_{\iota}\right)+p\left(\varphi_{\iota}\right) v\left(\varphi_{\iota}-\delta\right)\right)^{\prime}\right)+q_{2}\left(\varphi_{\iota}\right) g\left(v\left(\varphi_{\iota}-\varsigma\right)\right)=0, \quad \iota \in \mathbb{N} .
\end{array} .\right.
$$

In [6], Santra and Tripathy investigated the qualitative behavior of the impulsive system

$$
\left\{\begin{array}{l}
(v(\lambda)-p(\lambda) v(\lambda-\delta))^{\prime}+q(\lambda) g(v(\lambda-\varsigma))=0, \quad \lambda \neq \varphi_{\iota}, \quad \lambda \geq \lambda_{0} \\
v\left(\varphi_{l}^{+}\right)=I_{\iota}\left(v\left(\varphi_{\iota}\right)\right), \quad \iota \in \mathbb{N} \\
v\left(\varphi_{\iota}^{+}-\varsigma\right)=I_{\iota}\left(v\left(\varphi_{\iota}-\varsigma\right)\right), \quad \iota \in \mathbb{N}
\end{array}\right.
$$

for different values of the neutral coefficient $p$.

In [7], Santra and Dix obtained sufficient and necessary conditions for the oscillation of the impulsive system

$$
\left\{\begin{array}{l}
\left(r(\lambda)\left(f^{\prime}(\lambda)\right)^{\gamma}\right)^{\prime}+\sum_{j=1}^{m} q_{j}(\lambda) g_{j}\left(v\left(\tilde{s}_{j}(\lambda)\right)\right)=0, \quad \lambda \geq \lambda_{0}, \quad \lambda \neq \varphi_{\iota}, \quad \iota \in \mathbb{N} \\
\Delta\left(r\left(\varphi_{\iota}\right)\left(f^{\prime}\left(\varphi_{\iota}\right)\right)^{\gamma}\right)+\sum_{j=1}^{m} \widetilde{q}_{j}\left(\varphi_{\iota}\right) g_{j}\left(v\left(\tilde{\zeta}_{j}\left(\varphi_{\iota}\right)\right)\right)=0,
\end{array}\right.
$$

where

$$
f(\lambda)=v(\lambda)+p(\lambda) v(\delta(\lambda)), \quad \Delta v(\lambda)=\lim _{\eta \rightarrow \lambda^{+}} v(\eta)-\lim _{\eta \rightarrow \lambda^{-}} v(\eta), \quad-1 \leq p(\lambda) \leq 0 .
$$

In [8], Tripathy and Santra studied oscillatory behavior for the solutions of the following forced nonlinear neutral impulsive differential systems

$$
\left\{\begin{array}{l}
\left(r(\lambda)(u(\lambda)+p(\lambda) v(\lambda-\delta))^{\prime}\right)^{\prime}+q(\lambda) g(u(\lambda-\varsigma))=h(\lambda), \quad \lambda \neq \varphi_{\iota}, \quad \iota \in \mathbb{N} \\
\Delta\left(r\left(\varphi_{\iota}\right)\left(v\left(\varphi_{\iota}\right)+p\left(\varphi_{\iota}\right) v\left(\varphi_{\iota}-\delta\right)\right)^{\prime}\right)+\tilde{q}\left(\varphi_{l}\right) h\left(v\left(\varphi_{\iota}-\varsigma\right)\right)=\tilde{f}\left(\varphi_{\iota}\right), \quad \iota \in \mathbb{N}
\end{array}\right.
$$

for different values of $p(\lambda)$ and found some new sufficient conditions for the existence of positive bounded solutions of system (8).

For further details on recent developments of oscillation theory for delay differential equations and neutral impulsive differential equations, we refer the reader to the papers [9-36] and to the references therein. The study of qualitative behavior of halflinear/Emden-Fowler differential equations with deviating arguments has several applications in engineering and physics; see, e.g., the papers [26-29,31,32,34,35] for more details. In particular, by using different methods, the following papers were concerned with the qualitative behavior of various classes of half-linear/Emden-Fowler differential equation with different neutral coefficients (e.g., the paper [25] was concerned with neutral 
differential equations assuming that $0 \leq p(\lambda)<1$ and $p(\lambda)>1$; the paper [26] was concerned with neutral differential equations assuming that $0 \leq p(\lambda)<1$; the paper [28] was concerned with neutral differential equations assuming that $p(\lambda)$ is nonpositive; the papers $[29,33]$ were concerned with neutral differential equations in the case where $p(\lambda)>1$; the paper [32] was concerned with neutral differential equations assuming that $0 \leq p(\lambda) \leq q_{0}<\infty$ and $p(\lambda)>1$; the paper [34] was concerned with neutral differential equations in the case where $0 \leq p(\lambda) \leq q_{0}<\infty$; the paper [35] was concerned with neutral differential equations in the case when $0 \leq p(\lambda)=b_{0} \neq 1$; whereas the paper [31] was concerned with differential equations with a nonlinear neutral term assuming that $0 \leq p(\lambda) \leq a<1)$, which have the same research topic as that of this paper.

Motivated by above studies, in this article we establish new sufficient conditions for oscillation and non-oscillation properties of solutions to the following impulsive system

$$
\left\{\begin{array}{l}
{\left[r(\lambda)(v(\lambda)+p(\lambda) v(\delta(\lambda)))^{\prime}\right]^{\prime}+q(\lambda) G(v(\varsigma(\lambda)))=0, \lambda \neq \varphi_{\iota}, \iota \in \mathbb{N}} \\
\Delta\left[r\left(\varphi_{\iota}\right)\left(v\left(\varphi_{\iota}\right)+p\left(\varphi_{\iota}\right) v\left(\delta\left(\varphi_{\iota}\right)\right)\right)^{\prime}\right]+h\left(\varphi_{\iota}\right) G\left(v\left(\varsigma\left(\varphi_{\iota}\right)\right)\right)=0, \iota \in \mathbb{N} .
\end{array}\right.
$$

We suppose that following assumptions hols:

(A1) Let $\varphi_{\iota}, \iota \in \mathbb{N}$ with $\tau_{1}<\tau_{2}<\ldots<\varphi_{\iota}<\ldots$ and $\lim _{\iota \rightarrow \infty} \varphi_{\iota}=+\infty$ are fixed moment(i.e., a very brief period of time) of impulsive effect;

(A2) $\Delta$ is the difference operator defined by $\Delta V\left(\varphi_{l}\right)=V\left(\varphi_{l}+0\right)-V\left(\varphi_{l}-0\right)$;

(A3) $r \in \mathrm{C}\left(\left[\lambda_{0}, \infty\right),(0, \infty)\right)$ and $q, h \in \mathrm{C}\left(\left[\lambda_{0}, \infty\right),[0, \infty)\right)$, where $q$ and $h$ are not identically zero eventually;

(A4) $G \in \mathrm{C}(\mathbb{R}, \mathbb{R})$ is non-decreasing and satisfies $u G(u)>0$ for $u \neq 0$;

(A5) $\delta, \varsigma \in \mathrm{C}\left(\left[\lambda_{0}, \infty\right), \mathbb{R}\right)$ such that $\delta(\lambda), \varsigma(\lambda) \leq \lambda$ for $\lambda \geq \lambda_{0}, \delta(\lambda), \varsigma(\lambda) \rightarrow \infty$ as $\lambda \rightarrow \infty$ with differentiable and invertible $\delta$.

The investigation on the oscillatory or asymptotic behavior of solutions depend on the following two canonical and non-canonical conditions

(C1) $\int^{\infty} \frac{1}{r(\eta)} d \eta=\infty$ if and only if $\sum_{l=1}^{\infty} \frac{1}{r\left(\varphi_{l}\right)}=\infty$

and

(C2) $\int^{\infty} \frac{1}{r(\eta)} d \eta<\infty$ if and only if $\sum_{l=1}^{\infty} \frac{1}{r\left(\varphi_{l}\right)}<\infty$

respectively.

\section{Preliminary Results}

In this section, we present some lemmas for our further use. To simplify the notation, we set

$$
f(\lambda)=v(\lambda)+p(\lambda) v(\delta(\lambda))
$$

Lemma 1. Under the assumptions (C1), (A1)-(A5) and $v$ is an eventually positive solution of $(E)$ such that the companion function $f$ is also eventually positive, then $f$ satisfies

$$
f^{\prime}(\lambda)>0 \text { and }\left(r f^{\prime}\right)^{\prime}(\lambda)<0 \text { for all large } \lambda .
$$

Proof. Suppose that $v(\lambda)>0$ and $f(\lambda)>0$ for $\lambda \geq \lambda_{1}$, where $\lambda \geq \lambda_{0}$. By (A5), we may assume $v(\varsigma(\lambda))>0$ for $\lambda \geq \lambda_{1}$. By (E) and (A4), we have

$$
\begin{aligned}
\left(r f^{\prime}\right)^{\prime}(\lambda) & =-q(\lambda) G(v(\varsigma(\lambda)))<0 \\
\Delta\left(r f^{\prime}\right)\left(\varphi_{\iota}\right) & =-h\left(\varphi_{l}\right) G\left(v\left(\varsigma\left(\varphi_{\iota}\right)\right)\right)<0
\end{aligned}
$$

Consequently, $r f^{\prime}$ is non-increasing on $\left[\lambda_{1}, \infty\right)$ and thus either $f^{\prime}(\lambda)<0$ or $f^{\prime}(\lambda)>0$ for $\lambda \geq \lambda_{2}$, where $\lambda_{2} \geq \lambda_{1}$. If $f^{\prime}(\lambda)<0$, then for $\varepsilon>0$ we have $r(\lambda) f^{\prime}(\lambda) \leq-\varepsilon$ for 
$\lambda \geq \lambda_{2}$. A similar argument holds for the discrete equation, and we have $r\left(\varphi_{l}\right) f^{\prime}\left(\varphi_{l}\right) \leq-\varepsilon$. Integrating the relation $f^{\prime}(\lambda) \leq-\frac{\varepsilon}{r(\lambda)}$ over $\left[\lambda_{2}, \lambda\right) \subset\left[\lambda_{2}, \infty\right)$, we obtain

$$
f(\lambda)-f\left(\lambda_{2}\right)-\sum_{\lambda_{2} \leq \varphi_{l}<\lambda} f^{\prime}\left(\varphi_{l}\right) \leq-\varepsilon \int_{\lambda_{2}}^{\lambda} \frac{1}{r(\eta)} d \eta \quad \text { for } \lambda \geq \lambda_{2}
$$

that is,

$$
f(\lambda) \leq f\left(\lambda_{2}\right)-\varepsilon\left[\int_{\lambda_{2}}^{\lambda} \frac{1}{r(\eta)} d \eta+\sum_{\lambda_{2} \leq \varphi_{l}<\lambda} \frac{1}{r\left(\varphi_{l}\right)}\right] .
$$

In view of (C1), letting $\lambda \rightarrow \infty$ in (12) yields $f(\lambda) \rightarrow-\infty$, which is a contradiction. Therefore, $f^{\prime}(\lambda)>0$ for $\lambda \geq \lambda_{2}$. Thus, the lemma is proved.

Remark 1. It follows from Lemma 1 that $\lim _{\lambda \rightarrow \infty} f(\lambda)>0$, i.e., for $\varepsilon>0$ we have $f(\lambda) \geq \varepsilon$ for all large $\lambda$.

Lemma 2. Under the assumptions (C1), (A1)-(A5) and $v$ is an eventually positive solution of (E) such that the companion function $f$ is bounded, then $f$ satisfies (10) for all large $\lambda$.

\section{Oscillatory or Asymptotic Results}

In this section, we obtain some new sufficient conditions for the oscillatory and asymptotic behavior of solution of impulsive system (E). This section has been divided into two subsections depending on the canonical and non-canonical conditions (C1) and (C2).

\subsection{Oscillation under Canonical Condition}

In this subsection, we prove some new theorems on oscillatory or asymptotic behavior of solution of impulsive system (E) under the canonical condition (C1). Also, some examples are provided to validate the new results.

Theorem 1. Under the assumptions (C1), (A1)-(A5) and $0 \leq p(\lambda) \leq p<1$ for $\lambda \geq \lambda_{0}$, where $p$ is a real constant, and

(A6) $\int^{\infty} q(g) d g+\sum_{l=1}^{\infty} h\left(\varphi_{\iota}\right)=\infty$,

each solution of $(E)$ is oscillatory.

Proof. On the contrary, we assume that $v$ is a non-oscillatory solution of $(E)$. Therefore, for $\lambda_{1} \geq \lambda_{0}$ we have either $v(\lambda)<0$ or $v(\lambda)>0$ for $\lambda \geq \lambda_{1}$. First, we suppose that $v(\lambda)>0$, $v(\delta(\lambda))>0$ and $v(\varsigma(\lambda))>0$ for $\lambda \geq \lambda_{1}$. Clearly, $f$ defined by (9) is positive on $\left[\lambda_{1}, \infty\right)$. By Lemma 1 and Remark 1 , we get $\varepsilon \leq f(\lambda)$ for $\varepsilon>0$ and $\lambda \geq \lambda_{2}$, where $\lambda_{2} \geq \lambda_{1}$. Since $f$ is increasing, we have

$$
\begin{aligned}
(1-p) f(\lambda) & \leq(1-p(\lambda)) f(\lambda) \leq f(\lambda)-p(\lambda) f(\delta(\lambda)) \\
& =v(\lambda)-p(\lambda) p(\delta(\lambda)) v(\delta(\delta(\lambda))) \leq v(\lambda)
\end{aligned}
$$

for $\lambda \geq \lambda_{3}$, where $\lambda_{3} \geq \lambda_{2}$. Consequently, $v(\lambda) \geq(1-p) \varepsilon>0$ for $\lambda \geq \lambda_{3}$. From (11), we have

$$
\begin{aligned}
\left(r f^{\prime}\right)^{\prime}(\lambda)+G((1-p) \varepsilon) q(\lambda) & \leq 0 \\
\Delta\left(r f^{\prime}\right)\left(\varphi_{l}\right)+G((1-p) \varepsilon) h\left(\varphi_{l}\right) & \leq 0
\end{aligned}
$$

for $\lambda \geq \lambda_{3}$. Integrating the last system over the interval $\left[\lambda_{3}, \lambda\right) \subset\left[\lambda_{3}, \infty\right)$, we get

$$
G((1-p) \varepsilon) \int_{\lambda_{3}}^{\lambda} q(\eta) d \eta-\sum_{\lambda_{3} \leq \varphi_{l}<\lambda} \Delta\left(r f^{\prime}\right)\left(\varphi_{l}\right) \leq-\left[\left(r f^{\prime}\right)(\lambda)\right]_{\lambda_{3^{\prime}}}^{\lambda}
$$


that is,

$$
G((1-p) \varepsilon)\left[\int_{\lambda_{3}}^{\lambda} q(\eta) d \eta+\sum_{\lambda_{3} \leq \varphi_{l}<\lambda} h\left(\varphi_{l}\right)\right] \leq r\left(\lambda_{3}\right) f^{\prime}\left(\lambda_{3}\right)
$$

for $\lambda \geq \lambda_{3}$. This contradicts (A6). we find

If $v(\lambda)<0$ for $\lambda \geq \lambda_{1}$, then we set $y(\lambda):=-v(\lambda)$ for $\lambda \geq \lambda_{1}$ in (E). Using (A4),

$$
\left\{\begin{array}{l}
{\left[r(\lambda)(y(\lambda)+p(\lambda) y(\delta(\lambda)))^{\prime}\right]^{\prime}+q(\lambda) H(y(\varsigma(\lambda)))=0, \lambda \neq \varphi_{\iota}, \iota \in \mathbb{N}} \\
\Delta\left[r\left(\varphi_{\iota}\right)\left(y\left(\varphi_{\iota}\right)+p\left(\varphi_{\iota}\right) y\left(\delta\left(\varphi_{\iota}\right)\right)\right)^{\prime}\right]+h\left(\varphi_{\iota}\right) H\left(y\left(\varsigma\left(\varphi_{\iota}\right)\right)\right)=0, \iota \in \mathbb{N}
\end{array}\right.
$$

for $\lambda \geq \lambda_{1}$, where $H(v):=-G(-v)$ for $v \in \mathbb{R}$. Clearly, $H$ also satisfies (A4). Then, proceeding as as in the positive solution, we find the same contradiction.

Thus, the theorem is proved.

Theorem 2. Assume that (C1) and (A1)-(A5) hold, and $1 \leq p(\lambda) \leq p$ for $\lambda \geq \lambda_{0}$, where $p$ is a constant. If

(A7) there exists $\mu>0$ such that

$$
G(c)+G(d) \geq \mu G(c+d) \text { for } c, d \geq 0
$$

and

$$
G(c)+G(d) \leq \mu G(c+d) \text { for } c, d \leq 0 ;
$$

(A8) there exists $\mu>0$ such that

$$
G(c d) \leq G(c) G(d) \quad \text { for } c, d \geq 0
$$

and

$$
G(c d) \geq G(c) G(d) \quad \text { for } c, d \leq 0 ;
$$

(A9) $\delta(\varsigma(\lambda))=\varsigma(\delta(\lambda))$ for $\lambda \geq \lambda_{0}$;

(A10) $\int^{\infty} Q(\zeta) d \zeta+\sum_{l=1}^{\infty} H\left(\varphi_{l}\right)=\infty$, where

$$
Q(\zeta):=\min \left\{q(\zeta), q(\delta(\zeta)) \delta^{\prime}(\zeta)\right\} \quad \text { and } \quad H\left(\varphi_{\iota}\right):=\min \left\{h\left(\varphi_{l}\right), h\left(\delta\left(\varphi_{\iota}\right)\right) \delta\left(\varphi_{\iota}\right)\right\}
$$

hold for $\lambda \geq \lambda_{0}, \zeta \in \mathbb{R}$ and $\varphi_{\iota} \in \mathbb{R}$, then each solution of $(E)$ is oscillatory.

Proof. Proceeding as in the proof of Lemma 1, it is possible to show that $r f^{\prime}$ is nonincreasing and $f$ is monotonic on $\left[\lambda_{2}, \infty\right)$, where $\lambda_{2} \geq \lambda_{1}$. Clearly, $f$ defined by (9) is positive on $\left[\lambda_{1}, \infty\right)$. By Lemma 1 and Remark 1 , we get $\varepsilon \leq f(\lambda)$ for $\varepsilon>0$ and $\lambda \geq \lambda_{2}$, where $\lambda_{2} \geq \lambda_{1}$. Let us define

$$
w(\lambda):=r(\lambda) f^{\prime}(\lambda)+G(p) r(\delta(\lambda)) f^{\prime}(\delta(\lambda)) \text { for } \lambda \geq \lambda_{3},
$$

where $\lambda_{3} \geq \lambda_{2}$. From (E), we compute that

$$
\begin{aligned}
0 & =\left(r f^{\prime}\right)^{\prime}(\lambda)+q(\lambda) G(v(\varsigma(\lambda)))+G(p) \delta^{\prime}(\lambda)\left[\left(r f^{\prime}\right)^{\prime}(\delta(\lambda))+q(\delta(\lambda)) G(v(\varsigma(\delta(\lambda))))\right] \\
& =w^{\prime}(\lambda)+q(\lambda) G(v(\varsigma(\lambda)))+G(p) \delta^{\prime}(\lambda) q(\delta(\lambda)) G(v(\delta(\varsigma(\lambda))))
\end{aligned}
$$

for $\lambda \geq \lambda_{4}$, where $\lambda_{4} \geq \lambda_{3}$. Using (A7) and (A8), we obtain

$$
\begin{aligned}
0 & \geq w^{\prime}(\lambda)+Q(\lambda)[G(v(\varsigma(\lambda)))+G(p v(\delta(\varsigma(\lambda))))] \\
& \geq w^{\prime}(\lambda)+\mu Q(\lambda) G[v(\varsigma(\lambda))+p v(\delta(\varsigma(\lambda)))] \\
& \geq w^{\prime}(\lambda)+\mu Q(\lambda) G[v(\varsigma(\lambda))+p(\varsigma(\lambda)) v(\delta(\varsigma(\lambda)))] \\
& =w^{\prime}(\lambda)+\mu Q(\lambda) G(f(\varsigma(\lambda)))
\end{aligned}
$$


for $\lambda \geq \lambda_{4}$. Similarly, it is easy to find

$$
\Delta w\left(\varphi_{\iota}\right)+\mu H\left(\varphi_{\iota}\right) G\left(f\left(\varsigma\left(\varphi_{\iota}\right)\right)\right)=0
$$

for $\lambda \geq \lambda_{4}$. Consequently,

$$
\begin{aligned}
w^{\prime}(\lambda)+\mu Q(\lambda) G(\varepsilon) & \leq 0 \\
\Delta w\left(\varphi_{l}\right)+\mu H\left(\varphi_{l}\right) G(\varepsilon) & \leq 0
\end{aligned}
$$

for $\lambda \geq \lambda_{4}$, which upon integration over the interval $\left[\lambda_{4}, \lambda\right) \subset\left[\lambda_{4}, \infty\right)$ yields that

$$
\mu G(\varepsilon)\left[\int_{\lambda_{4}}^{\lambda} Q(\eta) d \eta+\sum_{\lambda_{4} \leq \varphi_{l}<\lambda} H\left(\varphi_{l}\right)\right] \leq w\left(\lambda_{4}\right) \quad \text { for all } \lambda \geq \lambda_{4} .
$$

This contradicts (A10). Thus, $v(\lambda)>0$ for $\lambda \geq \lambda_{1}$ cannot hold.

Thus, the proof is complete.

Remark 2. In Theorem 2, $\delta^{\prime}$ is allowed to be oscillatory

Let us give an important example for Theorem 2 where $\delta^{\prime}$ is allowed to be oscillatory.

Example 1. Consider the impulsive system

$$
\text { (E1) }\left\{\begin{array}{l}
{\left[v(\lambda)+2 v\left(\lambda-\sin \left(\frac{\pi}{2} \lambda\right)-1\right)\right]^{\prime \prime}+v(\lambda-4)=0, \lambda \neq \varphi_{\iota} \quad \iota \in \mathbb{N}} \\
\Delta\left[v\left(\varphi_{\iota}\right)+2 v\left(\varphi_{\iota}-\sin \left(\frac{\pi}{2} \varphi_{\iota}\right)-1\right)\right]^{\prime}+v\left(\varphi_{\iota}-4\right)=0, \quad \lambda \neq \varphi_{\iota \prime} \quad \iota \in \mathbb{N}
\end{array}\right.
$$

where $r(\lambda): \equiv 1, p(\lambda): \equiv 2, \delta(\lambda):=\lambda-\sin \left(\frac{\pi}{2} \lambda\right)-1, \varphi_{\iota}=\iota$ for $\iota \in \mathbb{N}, q(\lambda): \equiv 1: \equiv h\left(\varphi_{\iota}\right)$, $\varsigma(\lambda):=\lambda-4$ and $G(u):=u$ for $\lambda \geq 0$ and $u \in \mathbb{R}$. We have

$$
\delta(\varsigma(\lambda))=\lambda-\sin \left(\frac{\pi}{2} \lambda\right)-5=\varsigma(\delta(\lambda)) \quad \text { and } \quad \delta^{\prime}(\lambda)=1-\frac{\pi}{2} \cos \left(\frac{\pi}{2} \lambda\right) \quad \text { for } \quad \lambda \geq 0 .
$$

Note that $\delta^{\prime}$ is oscillatory and $Q(\lambda)=\min \left\{1,1-\frac{\pi}{2} \cos \left(\frac{\pi}{2} \lambda\right)\right\}$ for $\lambda \geq 0$. Obviously, $Q$ is a periodic function with a period 4 . Further, $\int_{0}^{4} Q(\eta) d \eta=2$, which shows that $\int^{\infty} Q(\eta) d \eta+$ $\sum_{l=1}^{\infty} H\left(\varphi_{l}\right)=\infty$. Then, by Theorem 2, every solution of (E1) oscillates.

Theorem 3. Under the assumptions (C1), (A1)-(A6) and $-1 \leq p(\lambda) \leq 0$ for $\lambda \geq \lambda_{0}$, each unbounded solution of $(E)$ is oscillatory.

Proof. Proceeding as in the proof of Lemma 1, it is possible to show that $r f^{\prime}$ is nonincreasing and $f$ is monotonic on $\left[\lambda_{2}, \infty\right)$, where $\lambda_{2} \geq \lambda_{1}$. Consequently, we have either $f(\lambda)<0$ or $f(\lambda)>0$ for $\lambda \geq \lambda_{2}$.

Case 1. Let $f(\lambda)<0$ for $\lambda \geq \lambda_{2}$. As $v$ is unbounded, for $\lambda \geq \lambda_{2}$ we have $v(\lambda)=\max \left\{v(\eta): \lambda_{2} \leq \eta \leq T\right\}$. Then, from (9), we have $v(\lambda) \leq f(\lambda)+v(\delta(\lambda))<v(\lambda)$, a contradiction.

Case 2. Consider $f(\lambda)>0$ for $\lambda \geq \lambda_{2}$. Using Lemma 1, (10) holds for $\lambda \geq \lambda_{3}$. Clearly, $f(\lambda) \leq v(\lambda)$ for $\lambda \geq \lambda_{3}$ implies

$$
\begin{aligned}
\left(r f^{\prime}\right)^{\prime}(\lambda)+q(\lambda) G(f(\varsigma(\lambda))) & \leq 0 \\
\Delta\left(r f^{\prime}\right)\left(\varphi_{\iota}\right)+h\left(\varphi_{\iota}\right) G(f(\varsigma(\lambda))) & \leq 0
\end{aligned}
$$


for $\lambda \geq \lambda_{3}$, where $\lambda_{4} \geq \lambda_{3}$. Further, by Lemma 1 and Remark 1 , we get $\varepsilon \leq f(\lambda)$ for $\varepsilon>0$ and $\lambda \geq \lambda_{4}$. Consequently, (15) becomes

$$
\begin{aligned}
\left(r f^{\prime}\right)^{\prime}(\lambda)+G(\varepsilon) q(\lambda) & \leq 0 \\
\Delta\left(r f^{\prime}\right)\left(\varphi_{\iota}\right)+G(\varepsilon) h\left(\varphi_{\iota}\right) & \leq 0
\end{aligned}
$$

for $\lambda \geq \lambda_{4}$. Integrating the last inequality over $\left[\lambda_{4}, \lambda\right) \subset\left[\lambda_{4}, \infty\right)$, we have

$$
G(\varepsilon)\left[\int_{\lambda_{4}}^{\lambda} q(\eta)+\sum_{\lambda_{4} \leq \varphi_{l}<\lambda} h\left(\varphi_{\iota}\right)\right] d \eta \leq r\left(\lambda_{4}\right) f^{\prime}\left(\lambda_{4}\right) \quad \text { for } \lambda \geq \lambda_{4}
$$

This contradicts (A6).

This completes the proof.

Theorem 4. Under the assumptions (C1), (A1)-(A6) and $-1<-p \leq p(\lambda) \leq 0$ for $\lambda \geq \lambda_{0}$, every bounded solution of $(E)$ is either oscillatory or $\lim _{\lambda \rightarrow \infty} v(\lambda)=0$.

Proof. Proceeding as in the proof of Lemma 2, it is possible to show that $r f^{\prime}$ is nonincreasing and $f$ is monotonic on $\left[\lambda_{2}, \infty\right)$, where $\lambda_{2} \geq \lambda_{1}$. Consequently, we have either $f(\lambda)<0$ or $f(\lambda)>0$ for $\lambda \geq \lambda_{2}$.

Case 1. Consider $f(\lambda)<0$ for $\lambda \geq \lambda_{2}$. Then, $\lim _{\lambda \rightarrow \infty} f(\lambda)$ exists. Thus, we have

$$
\begin{aligned}
& 0 \geq \lim _{\lambda \rightarrow \infty} f(\lambda)=\limsup _{\lambda \rightarrow \infty} f(\lambda)=\limsup _{\lambda \rightarrow \infty}[v(\lambda)+p(\lambda) v(\delta(\lambda))] \geq \limsup _{\lambda \rightarrow \infty}[v(\lambda)-p v(\delta(\lambda))] \\
& \geq \limsup _{\lambda \rightarrow \infty} v(\lambda)+\liminf _{\lambda \rightarrow \infty}[-p v(\delta(\lambda))]=(1-p) \limsup _{\lambda \rightarrow \infty} v(\lambda)
\end{aligned}
$$

i.e., $\lim \sup _{\lambda \rightarrow \infty} v(\lambda)=0$ (since $0<p<1$ ) and hence $\lim _{\lambda \rightarrow \infty} v(\lambda)=0$ for $\lambda \neq \varphi_{\iota}$ where $\iota \in \mathbb{N}$. We note that $\left\{v\left(\varphi_{l}-0\right)\right\}_{l=1}^{\infty}$ and $\left\{v\left(\varphi_{l}+0\right)\right\}_{l=1}^{\infty}$ are sequences of reals. Therefore, $\liminf _{\lambda \rightarrow \infty} v(\lambda)=0$ and $\lim \sup _{\lambda \rightarrow \infty} v(\lambda)=0$ coincide with $\lim _{\iota \rightarrow \infty} v\left(\varphi_{\iota}-0\right)=0$ and $\lim _{\iota \rightarrow \infty} v\left(\varphi_{\iota}+0\right)=0$ respectively. As a result, $\lim _{\lambda \rightarrow \infty} v(\lambda)=0$ for all $\lambda$ and $\varphi_{\iota}$ where $\iota \in \mathbb{N}$.

Case 2. Consider $f(\lambda)>0$ for $\lambda \geq \lambda_{2}$. This case follows from Theorem 3 .

Thus, the proof is complete.

Corollary 1. Under the assumptions (C1), (A1)-(A6) and $-1<-p \leq p(\lambda) \leq 0$ for $\lambda \geq \lambda_{0}$, where $p$ is a constant, every solution of $(E)$ is either oscillatory or $\lim _{\lambda \rightarrow \infty} v(\lambda)=0$.

Theorem 5. Under the assumptions (C1), (A1)-(A6) and $-p_{1} \leq p(\lambda) \leq-p_{2}<-1$ for $\lambda \geq \lambda_{0}$, every bounded solution of $(E)$ is either oscillatory or $\lim _{\lambda \rightarrow \infty} v(\lambda)=0$.

Proof. Proceeding as in the proof of Lemma 2, it is possible to show that $r f^{\prime}$ is nonincreasing and $f$ is monotonic on $\left[\lambda_{2}, \infty\right)$, where $\lambda_{2} \geq \lambda_{1}$. Consequently, we have either $f(\lambda)<0$ or $f(\lambda)>0$ for $\lambda \geq \lambda_{2}$.

Case 1. Assume that $f(\lambda)<0$ for $\lambda \geq \lambda_{2}$. In this case, $\lim _{\lambda \rightarrow \infty} f(\lambda)$ exists as a nonpositive finite value. We claim that $\lim _{\lambda \rightarrow \infty} f(\lambda)=0$. Otherwise, $\lim _{\lambda \rightarrow \infty} f(\lambda)<0$, i.e., for $\varepsilon>0$ we have $-\varepsilon>f(\lambda)$ for $\lambda \geq \lambda_{1}$. Hence, $f(\lambda) \geq p(\lambda) v(\delta(\lambda)) \geq-p_{1} v(\delta(\lambda))$ for $\lambda \geq \lambda_{1}$, which implies $v(\lambda) \geq-\frac{1}{p_{1}} f\left(\delta^{-1}(\lambda)\right) \geq \frac{\varepsilon}{p_{1}}$ for $\lambda \geq \lambda_{1}$. Consequently, (11) becomes

$$
\begin{aligned}
\left(r f^{\prime}\right)^{\prime}(\lambda)+q(\lambda) G\left(\frac{\varepsilon}{p_{1}}\right) & \leq 0 \\
\Delta\left(r f^{\prime}\right)\left(\varphi_{\iota}\right)+h\left(\varphi_{\iota}\right) G\left(\frac{\varepsilon}{p_{1}}\right) & \leq 0
\end{aligned}
$$


for $\lambda \geq \lambda_{1}$. Integrating the last inequality over the interval $\left[\lambda_{1}, \lambda\right) \subset\left[\lambda_{1}, \infty\right)$, we get

$$
G\left(\frac{\varepsilon}{p_{1}}\right)\left[\int_{\lambda_{2}}^{\lambda} q(\eta) d \eta+\sum_{\lambda_{2} \leq \varphi_{l}<\lambda} h\left(\varphi_{l}\right)\right] \leq r\left(\lambda_{2}\right) f^{\prime}\left(\lambda_{2}\right) \quad \text { for } \lambda \geq \lambda_{1} .
$$

This contradicts (A6). Therefore, $\lim _{\lambda \rightarrow \infty} f(\lambda)=0$. Hence,

$$
\begin{aligned}
0 & =\lim _{\lambda \rightarrow \infty} f(\lambda)=\liminf _{\lambda \rightarrow \infty} f(\lambda) \leq \liminf _{\lambda \rightarrow \infty}\left[v(\lambda)-p_{2} v(\delta(\lambda))\right] \\
& \leq \limsup _{\lambda \rightarrow \infty} v(\lambda)+\liminf _{\lambda \rightarrow \infty}\left[-p_{2} v(\delta(\lambda))\right] \leq\left(1-p_{2}\right) \limsup _{\lambda \rightarrow \infty} v(\lambda),
\end{aligned}
$$

which shows that $\lim \sup _{\lambda \rightarrow \infty} v(\lambda)=0$ due to $p_{2}>1$. Thus, $\liminf _{\lambda \rightarrow \infty} v(\lambda)=0$ and hence $\lim _{\lambda \rightarrow \infty} v(\lambda)=0$. Therefore, any non-oscillatory solution $v$ of (E) converges to zero.

Case 2. Assume that $f(\lambda)>0$ for $\lambda \geq \lambda_{2}$. The case follows from Theorem 3 .

Thus, the theorem is proved.

Example 2. Consider the impulsive system

$$
\text { (E2) }\left\{\begin{array}{l}
{\left[\lambda\left(v(\lambda)-3 v\left(e^{-\pi} \lambda\right)\right)^{\prime}\right]^{\prime}+\frac{4}{\lambda} v(\lambda)=0, \lambda \neq \varphi_{\iota}, \quad \iota \in \mathbb{N} \text { for } \quad \lambda \geq 1,} \\
\Delta\left[\varphi_{\iota}\left(v(\lambda)-3 v\left(e^{-\pi} \varphi_{\iota}\right)\right)^{\prime}\right]+\frac{4}{2 \iota} v\left(\varphi_{\iota}\right)=0, \quad \lambda \neq \varphi_{\iota}, \quad \iota \in \mathbb{N}
\end{array}\right.
$$

where $r(\lambda):=\lambda, p(\lambda): \equiv-3, \delta(\lambda):=e^{-\pi} \lambda, q(\lambda):=\frac{4}{\lambda}, \varphi_{\iota}=2 \iota$ for $\iota \in \mathbb{N}, h\left(\varphi_{\iota}\right)=\frac{4}{2}$, $\varsigma(\lambda):=\lambda$ and $G(u):=u$ for $\lambda \geq 1$ and $u \in \mathbb{R}$. It can be easily shown that Theorem 5 applies to (E2). Thus, every bounded solution oscillates or $\lim _{\lambda \rightarrow \infty} v(\lambda)=0$. Obviously, $v(\lambda)=\sin (\ln (\lambda))$ for $\lambda \geq 1$ is an oscillating solution.

\subsection{Oscillation under Non-Canonical Conditions}

In this subsection, we are proving some new theorems on oscillatory or asymptotic behavior of the solution of impulsive system (E) under the non-canonical condition (C2). Also, one examples is provided to validate the new results.

Remark 3. Letting

$$
R(\lambda):=\int_{\lambda}^{\infty} \frac{1}{r(\zeta)} d \zeta \text { for } \lambda \geq \lambda_{0}
$$

(C2) implies that $R(\lambda) \rightarrow 0$ as $\lambda \rightarrow \infty$.

Lemma 3. Under the assumptions (C2), (A1)-(A5) and $v$ is an eventually positive solution of $(E)$ such that the companion function $f$ is eventually decreasing and positive, then we have

$$
\varepsilon R(\lambda) \leq f(\lambda) \text { for all large } \lambda \text { and } \varepsilon>0,
$$

where $R$ is defined in (16).

Proof. Suppose that $f(\lambda), v(\lambda)>0$ and $f^{\prime}(\lambda)<0$ for $\lambda \geq \lambda_{1}$, where $\lambda \geq \lambda_{0}$. By (A5), we can assume $v(\varsigma(\lambda))>0$ for $\lambda \geq \lambda_{1}$. From (E) and (A4), we get (11). Consequently, $r f^{\prime}$ is non-increasing on $\left[\lambda_{1}, \infty\right)$. Therefore, $r(s) f^{\prime}(s) \leq r(\lambda) f^{\prime}(\lambda)$ for $s \geq \lambda \geq \lambda_{1}$, which implies

$$
f^{\prime}(s) \leq \frac{r(\lambda) f^{\prime}(\lambda)}{r(s)} \text { for } s \geq \lambda \geq \lambda_{1} .
$$

Consequently,

$$
f(s) \leq f(\lambda)+r(\lambda) f^{\prime}(\lambda) \int_{\lambda}^{s} \frac{1}{r(\eta)} d \eta \quad \text { for } s \geq \lambda \geq \lambda_{1} .
$$


As $r f^{\prime}$ is non-increasing, we can find a constant $\varepsilon>0$ such that $r(\lambda) f^{\prime}(\lambda) \leq-\varepsilon$ for $\lambda \geq \lambda_{1}$. As a result $f(s) \leq f(\lambda)-\varepsilon \int_{\lambda}^{s} \frac{1}{r(\eta)} d \eta$ for $s \geq \lambda \geq \lambda_{1}$. By letting $s \rightarrow \infty$, we get $0 \leq f(\lambda)-\varepsilon R(\lambda)$ for $\lambda \geq \lambda_{1}$, which proves (17).

Theorem 6. Under the assumptions (C2), (A1)-(A5), (A7)-(A10) and $0 \leq p(\lambda) \leq p$ for $\lambda \geq \lambda_{0}$, where $p$ is a constant, and

(A11)

$$
\int_{\lambda_{0}}^{\infty} \frac{1}{r(\eta)} \int_{\lambda_{0}}^{\eta} Q(\zeta) G(\varepsilon R(\varsigma(\zeta))) d \zeta d \eta+R\left(\lambda_{0}\right) \sum_{l=1}^{\infty} H\left(\varphi_{l}\right) G\left(\varepsilon R\left(\varsigma\left(\varphi_{\iota}\right)\right)\right)=\infty
$$

for every $\varepsilon>0$, and

$$
\int_{\lambda_{0}}^{\infty} \frac{1}{r(\eta)} \int_{\lambda_{0}}^{\eta} Q(\zeta) G(\varepsilon R(\varsigma(\zeta))) d \zeta d \eta+R\left(\lambda_{0}\right) \sum_{l=1}^{\infty} H\left(\varphi_{l}\right) G\left(\varepsilon R\left(\zeta\left(\varphi_{l}\right)\right)\right)=-\infty
$$

for every $\varepsilon<0$, where $Q$ and $H$ are defined in (A10), each solution of $(E)$ is oscillatory.

Proof. Proceeding as in the proof of Lemma 1 we obtain (11) for $\lambda \geq \lambda_{1}$, i.e., $r f^{\prime}$ is nonincreasing on $\left[\lambda_{2}, \infty\right)$, where $\lambda_{2} \geq \lambda_{1}$. Note that $f$ is positive on $\left[\lambda_{2}, \infty\right)$. Consequently, we have either $f^{\prime}(\lambda)<0$ or $f^{\prime}(\lambda)>0$ for $\lambda \geq \lambda_{2}$.

Case 1. Assume that $f^{\prime}(\lambda)<0$ for $\lambda \geq \lambda_{2}$. From Lemma 3, we have (17) for $\lambda \geq \lambda_{3}$, where $\varepsilon>0$ and $\lambda_{3} \geq \lambda_{2}$. Using (17) in (13) and (14), we have

$$
\begin{aligned}
w^{\prime}(\lambda)+\mu Q(\lambda) G(\varepsilon R(\varsigma(\lambda))) & \leq 0 \\
\Delta w\left(\varphi_{\iota}\right)+\mu H\left(\varphi_{\iota}\right) G\left(\varepsilon R\left(\varsigma\left(\varphi_{\iota}\right)\right)\right) & \leq 0
\end{aligned}
$$

for $\lambda \geq \lambda_{3}$, where $\lambda_{3} \geq \lambda_{2}$. Integrating the last inequality over the interval $\left[\lambda_{3}, \lambda\right) \subset\left[\lambda_{3}, \infty\right)$, we obtain

$$
\begin{aligned}
\mu\left[\int_{\lambda_{3}}^{\lambda} Q(\eta) G(\varepsilon R(\varsigma(\eta))) d \eta+\sum_{\lambda_{3} \leq \varphi_{l}<\lambda} H\left(\varphi_{l}\right) G\left(\varepsilon R\left(\varsigma\left(\varphi_{l}\right)\right)\right)\right] & \leq-w(\lambda) \\
& \leq-(1+G(p)) r(\lambda) f^{\prime}(\lambda)
\end{aligned}
$$

which implies

$$
\frac{\mu}{1+G(p)} \frac{1}{r(\lambda)}\left[\int_{\lambda_{3}}^{\lambda} Q(\eta) G(\varepsilon R(\varsigma(\eta))) d \eta+\sum_{\lambda_{3} \leq \varphi_{l}<\lambda} H\left(\varphi_{\iota}\right) G\left(\varepsilon R\left(\varsigma\left(\varphi_{\iota}\right)\right)\right)\right] \leq-f^{\prime}(\lambda)
$$

for $\lambda \geq \lambda_{3}$. Again integrating the last inequality over the interval $\left[\lambda_{3}, \lambda\right) \subset\left[\lambda_{3}, \infty\right)$, we obtain

$$
\begin{aligned}
\frac{\mu}{1+G(p)} \int_{\lambda_{3}}^{\lambda} \frac{1}{r(\eta)} & {\left[\int_{\lambda_{3}}^{\eta} Q(\zeta) G(\varepsilon R(\varsigma(\zeta))) d \zeta+\sum_{\lambda_{3} \leq \varphi_{l}<\lambda} H\left(\varphi_{l}\right) G\left(\varepsilon R\left(\varsigma\left(\varphi_{l}\right)\right)\right)\right] d \eta } \\
& \leq-[f(\eta)]_{\lambda_{3}}^{\lambda}+\sum_{\lambda_{3} \leq \varphi_{l}<\lambda} \Delta f\left(\varphi_{l}\right) \\
& =-[f(\eta)]_{\lambda_{3}}^{\lambda}+\sum_{\lambda_{3} \leq \varphi_{l}<\lambda}\left[f\left(\varphi_{l}+0\right)-f\left(\varphi_{l}-0\right)\right] \\
& \leq f\left(\lambda_{3}\right)+\sum_{\lambda_{3} \leq \varphi_{l}<\lambda} f\left(\varphi_{l}+0\right)
\end{aligned}
$$

which contradicts (A11).

Case 2. Assume that $f^{\prime}(\lambda)>0$ for $\lambda \geq \lambda_{2}$. The case follows from the proof of Theorem 2.

Thus, the theorem is proved. 
Example 3. Consider the impulsive systems

$$
\text { (E3) }\left\{\begin{array}{l}
{\left[e^{\lambda}\left(v(\lambda)+3 e^{-\lambda} v(\lambda-3)\right)^{\prime}\right]^{\prime}+e^{3 t}(v(\lambda-1))^{3}=0, \quad \lambda \neq \iota, \quad \iota \in \mathbb{N}} \\
\Delta\left[e^{\iota}\left(v\left(\varphi_{\iota}\right)+3 e^{-\iota} v\left(\varphi_{\iota}-3\right)\right)^{\prime}\right]+e^{3 \iota}\left(v\left(\varphi_{\iota}-1\right)\right)^{3}=0, \quad \iota \in \mathbb{N}
\end{array}\right.
$$

for $\lambda \geq 3$, where $r(\lambda):=e^{\lambda}, R(\lambda):=e^{-\lambda}, p(\lambda):=3 e^{-\lambda}, \delta(\lambda):=\lambda-3, q(\lambda):=e^{3 \lambda}, \varphi_{\iota}=\iota$ for $\iota \in \mathbb{N}, h\left(\varphi_{\iota}\right)=e^{3 \iota}, \varsigma(\lambda):=\lambda-1$ and $G(u):=u^{3}$ for $\lambda \geq 3$ and $u \in \mathbb{R}$. Therefore, by Theorem 6 , every solution of (E3) oscillates.

Theorem 7. Assume that (C2), (A1)-(A6), $-1 \leq p(\lambda) \leq 0$ for $\lambda \geq \lambda_{0}$, and

$$
\int_{\lambda_{0}}^{\infty} \frac{1}{r(\eta)} \int_{\lambda_{0}}^{\eta} q(\zeta) G(\varepsilon R(\zeta(\zeta))) d \zeta d \eta+R\left(\lambda_{0}\right) \sum_{l=1}^{\infty} h\left(\varphi_{\iota}\right) G\left(\varepsilon R\left(\zeta\left(\varphi_{\iota}\right)\right)\right)=\infty
$$

for every $\varepsilon>0$, and

$$
\int_{\lambda_{0}}^{\infty} \frac{1}{r(\eta)} \int_{\lambda_{0}}^{\eta} q(\zeta) G(\varepsilon R(\zeta(\zeta))) d \zeta d \eta+R\left(\lambda_{0}\right) \sum_{\iota=1}^{\infty} h\left(\varphi_{\iota}\right) G\left(\varepsilon R\left(\varsigma\left(\varphi_{\iota}\right)\right)\right)=-\infty
$$

for every $\varepsilon<0$,

every unbounded solution of $(E)$ oscillates.

Proof. Proceeding as in the proof of Lemma 1, it is possible to show that $f$ and $f^{\prime}$ are of single sign on $\left[\lambda_{2}, \infty\right)$, where $\lambda_{2} \geq \lambda_{1}$. Consequently, we have either $f(\lambda)<0$ or $f(\lambda)>0$ for $\lambda \geq \lambda_{2}$.

Case 1. Let $f(\lambda)<0$ for $\lambda \geq \lambda_{2}$. The proof is similar to Case 2 in the proof of Theorem 3.

Case 2. Let $f(\lambda)>0$ for $\lambda \geq \lambda_{2}$. Note that $v(\lambda) \geq f(\lambda)$ for $\lambda \geq \lambda_{2}$.

(a) Assume that $f^{\prime}(\lambda)>0$ for $\lambda \geq \lambda_{2}$. We easily get (15). Then, proceeding as in Case 1 in the proof of Theorem 3, we get a contradiction.

(b) Assume that $f^{\prime}(\lambda)<0$ for $\lambda \geq \lambda_{2}$. From Lemma 3, we have (17) for $\lambda \geq \lambda_{3}$, where $\varepsilon>0$ and $\lambda_{3} \geq \lambda_{2}$. Using $f(\lambda) \leq v(\lambda)$ for $\lambda \geq \lambda_{2}$ and (11), we get

$$
\begin{aligned}
\left(r f^{\prime}\right)^{\prime}(\lambda)+q(\lambda) G(\varepsilon R(\varsigma(\lambda))) & \leq 0 \\
\Delta\left(r f^{\prime}\right)\left(\varphi_{\iota}\right)+h\left(\varphi_{\iota}\right) G\left(\varepsilon R\left(\varsigma\left(\varphi_{\iota}\right)\right)\right) & \leq 0
\end{aligned}
$$

for $\lambda \geq \lambda_{3}$, where $\lambda_{3} \geq \lambda_{2}$. The remaining proof is similar to Case 2 of Theorem 6 .

The proof is therefore completed.

Theorem 8. Under the assumptions (C2), (A1)-(A6), (A12) and $-1<-p \leq p(\lambda) \leq 0$ for $\lambda \geq \lambda_{0}$, every bounded solution of $(E)$ is either oscillatory or $\lim _{\lambda \rightarrow \infty} v(\lambda)=0$.

Proof. Proceeding as in the proof of Lemma 1, it is possible to show that $f$ and $f^{\prime}$ are of single sign on $\left[\lambda_{2}, \infty\right)$, where $\lambda_{2} \geq \lambda_{1}$. Consequently, we have the following two possible cases.

Case 1. Let $f(\lambda)<0$ for $\lambda \geq \lambda_{2}$. Recalling that $f$ is monotonic, we follow the steps in Case 2 in the proof of Theorem 4 and see that $\lim _{\lambda \rightarrow \infty} v(\lambda)=0$.

Case 2. Assume $f(\lambda)>0$ for $\lambda \geq \lambda_{2}$. The case follows from Case 1 of Theorem 7 .

This completes the proof of the theorem.

Corollary 2. Under the assumptions (C2), (A1)-(A6), (A12) and $-1<-p \leq p(\lambda) \leq 0$ for $\lambda \geq \lambda_{0}$, where $p$ is a constant, every solution of $(E)$ is either oscillatory or $\lim _{\lambda \rightarrow \infty} \nu(\lambda)=0$.

Theorem 9. Under the assumptions (C2), (A1)-(A6), (A12) and $-p_{1} \leq p(\lambda) \leq-p_{2}<-1$ for $\lambda \geq \lambda_{0}$, where $p_{1}$ and $p_{2}$ are constants, and 
$(A 13) \int_{\lambda_{0}}^{\infty} \frac{1}{r(\eta)} \int_{\lambda_{0}}^{\eta} q(\zeta) d \zeta d \eta+R\left(\lambda_{0}\right) \sum_{l=1}^{\infty} h\left(\varphi_{\iota}\right)=\infty$,

every bounded solution of $(E)$ is either oscillatory or $\lim _{\lambda \rightarrow \infty} v(\lambda)=0$.

Proof. Proceeding as in the proof of Lemma 1, it is possible to show that $f$ and $f^{\prime}$ are of single sign on $\left[\lambda_{2}, \infty\right)$, where $\lambda_{2} \geq \lambda_{1}$. Consequently, we have the following two possible cases.

Case 1. Let $f(\lambda)<0$ for $\lambda \geq \lambda_{2}$. In this case, $\lim _{\lambda \rightarrow \infty} f(\lambda)$ exists as a non-positive finite value. We claim that $\lim _{\lambda \rightarrow \infty} f(\lambda)=0$. Otherwise, $\lim _{\lambda \rightarrow \infty} f(\lambda)<0$, i.e., for $\varepsilon>0$ we have $f(\lambda)<-\varepsilon$ for $\lambda \geq \lambda_{2}$. Then, we have $f(\lambda) \geq p(\lambda) v(\delta(\lambda)) \geq-p_{1} v(\delta(\lambda))$ for $\lambda \geq \lambda_{2}$, which implies $v(\lambda) \geq-\frac{1}{p_{1}} f\left(\delta^{-1}(\lambda)\right) \geq \frac{\varepsilon}{p_{1}}$ for $\lambda \geq \lambda_{2}$. Consequently, (11) becomes

$$
\begin{aligned}
\left(r f^{\prime}\right)^{\prime}(\lambda)+q(\lambda) G\left(\frac{\varepsilon}{p_{1}}\right) & \leq 0 \\
\Delta\left(r f^{\prime}\right)\left(\varphi_{\iota}\right)+h\left(\varphi_{\iota}\right) G\left(\frac{\varepsilon}{p_{1}}\right) & \leq 0
\end{aligned}
$$

for $\lambda \geq \lambda_{2}$. Integrating the last inequality over the interval $\left[\lambda_{2}, \lambda\right) \subset\left[\lambda_{2}, \infty\right)$, we get

$$
\left[\left(r f^{\prime}\right)(\eta)\right]_{\lambda_{2}}^{\lambda}-\sum_{\lambda_{2} \leq \varphi_{l}<\lambda} \Delta\left(r f^{\prime}\right)\left(\varphi_{l}\right)+G\left(\frac{\varepsilon}{p_{1}}\right) \int_{\lambda_{2}}^{\lambda} q(\eta) d \eta \leq 0,
$$

that is,

$$
-r\left(\lambda_{2}\right) f^{\prime}\left(\lambda_{2}\right)+G\left(\frac{\varepsilon}{p_{1}}\right)\left[\int_{\lambda_{2}}^{\lambda} q(\eta) d \eta+\sum_{\lambda_{2} \leq \varphi_{l}<\lambda} h\left(\varphi_{l}\right)\right] \leq-r(\lambda) f^{\prime}(\lambda) \quad \text { for } \lambda \geq \lambda_{2}
$$

Again, integrating the last inequality over the interval $\left[\lambda_{2}, \lambda\right) \subset\left[\lambda_{2}, \infty\right)$ after dividing through by $r$, we get

$$
\begin{aligned}
-r\left(\lambda_{2}\right) f^{\prime}\left(\lambda_{2}\right) \int_{\lambda_{2}}^{\lambda} \frac{1}{r(\eta)} d \eta & +G\left(\frac{\varepsilon}{p_{1}}\right) \int_{\lambda_{2}}^{\lambda} \frac{1}{r(\eta)}\left[\int_{\lambda_{2}}^{\eta} q(\zeta) d \zeta+\sum_{\lambda_{2} \leq \varphi_{l}<\lambda} h\left(\varphi_{\iota}\right)\right] d \eta \\
& \leq-[f(\eta)]_{\lambda_{2}}^{\lambda}+\sum_{\lambda_{2} \leq \varphi_{l}<\lambda} \Delta f\left(\varphi_{\iota}\right) \\
& \leq-f(\lambda)-\sum_{\lambda_{2} \leq \varphi_{\iota}<\lambda} f\left(\varphi_{\iota}-0\right)
\end{aligned}
$$

for $\lambda \geq \lambda_{2}$, which contradicts $(A 13)$ by (C2). Therefore, $\lim _{\lambda \rightarrow \infty} f(\lambda)=0$. For the rest of the proof, we follow the steps in the last part of Case 2 of Theorem 5 to get $\lim _{\lambda \rightarrow \infty} v(\lambda)=0$.

Case 2. Assume $f(\lambda)>0$ for $\lambda \geq \lambda_{2}$. In this case, we proceed as in Case 1 in the proof of Theorem 7 and get a contradiction.

Hence, the theorem is proved.

\section{Conclusions and Open Problem}

In this paper, we established some new sufficient conditions for the qualitative behavior of the solution of the impulsive system under the canonical and non-canonical conditions (C1) and (C2) for $|p(\lambda)|<+\infty$. In addition, some illustrated examples are provided to verify main results. It would be of interest to examine the oscillation of $(\mathrm{E})$ with different neutral coefficients; see, e.g., the papers [25,28,29,32-35] for more details. Furthermore, it is also interesting to analyze the oscillation of (E) with a nonlinear neutral term; see, e.g., the paper [31] for more details.

Author Contributions: Conceptualization, S.S.S., K.M.K., K.N. and H.A.; methodology, S.S.S., K.M.K., K.N. and H.A.; validation, S.S.S., K.M.K., K.N. and H.A.; formal analysis, S.S.S., K.M.K., K.N. and H.A.; investigation, S.S.S., K.M.K., K.N. and H.A.; writing-review and editing, S.S.S., K.M.K., K.N. 
and H.A.; supervision, S.S.S., K.M.K., K.N. and H.A.; funding acquisition, K.M.K. and K.N. All authors have read and agreed to the published version of the manuscript.

Funding: Deanship of Scientific Research at King Khalid University for funding this work through the small research groups under grant number RGP. 1/372/42.

Institutional Review Board Statement: Not applicable.

Informed Consent Statement: Not applicable.

Data Availability Statement: Not applicable.

Acknowledgments: The Authors extend their thanks to the Deanship of Scientific Research at King Khalid University for funding this work through the small research groups under grant number RGP. $1 / 372 / 42$.

Conflicts of Interest: The authors declare no conflict of interest.

\section{References}

1. Shen, J.H.; Wang, Z.C. Oscillation and asympotic behaviour of solutions of delay differential equations with impulses. Ann. Differ. Eqs. 1994, 10, 61-68.

2. Graef, J.R.; Shen, J.H.; Stavroulakis, I.P. Oscillation of impulsive neutral delay differential equations. J. Math. Anal. Appl. 2002, 268, 310-333. [CrossRef]

3. Shen, J.; Zou, Z. Oscillation criteria for first order impulsive differential equations with positive and negative coefficients. J. Comput. Appl. Math. 2008, 217, 28-37. [CrossRef]

4. Tripathy, A.K.; Santra, S.S. Characterization of a class of second-order neutral impulsive systems via pulsatile constant. Differ. Equ. Appl. 2017, 9, 87-98. [CrossRef]

5. Tripathy, A.K.; Santra, S.S. Necessary and Sufficient Conditions for Oscillation of a Class of second-order Impulsive Systems. Differ. Equ. Dyn. Syst. 2018. [CrossRef]

6. Santra, S.S.; Tripathy, A.K. On oscillatory first order nonlinear neutral differential equations with nonlinear impulses. J. Appl. Math. Comput. 2019, 59, 257-270. [CrossRef]

7. Santra, S.S.; Dix, J.G. Necessary and sufficient conditions for the oscillation of solutions to a second-order neutral differential equation with impulses. Nonlinear Stud. 2020, 27, 375-387. [CrossRef]

8. Tripathy, A.K.; Santra, S.S. On the forced impulsive oscillatory nonlinear neutral systems of the second-order. Nonlinear Oscil. 2020, 23, 274-288.

9. Bazighifan, O.; Scapellato, A. Oscillatory properties of even-order ordinary differential equations with variable coefficients. Miskolc Math. Notes 2020, 21, 641-652. [CrossRef]

10. Santra, S.S.; Ghosh, T.; Bazighifan, O. Explicit Criteria for the Oscillation of Second-Order Differential Equations with Several Sub-linear Neutral Coefficients. Adv. Differ. Equ. 2020, 643. [CrossRef]

11. Santra, S.S.; Dassios, I.; Ghosh, T. On the asymptotic behavior of a class of second-order non-linear neutral differential Equations with multiple delays. Axioms 2020, 9, 134. [CrossRef]

12. Santra, S.S.; Majumder, D.; Bhattacharjee, R.; Bazighifan, O.; Khedher, K.; Marin, M. New Theorems for Oscillations to the Differential Equations with Mixed Delays. Symmetry 2021, 13, 367. [CrossRef]

13. Santra, S.S.; Bazighifan, O.; Ahmad, H.; Chu, Y.-M. Second-Order Differential Equation: Oscillation Theorems and Applications. Math. Probl. Eng. 2020, 2020. [CrossRef]

14. Santra, S.S.; Bazighifan, O.; Ahmad, H.; Yao, S.-W. Second-Order Differential Equation with Multiple Delays: Oscillation Theorems and Applications. Complexity 2020, 2020, 8853745. [CrossRef]

15. Bazighifan, O.; Ruggieri, M.; Scapellato, A. An Improved Criterion for the Oscillation of Fourth-Order Differential Equations. Mathematics 2020, 8, 610. [CrossRef]

16. Bazighifan, O.; Ruggieri, M.; Santra, S.S.; Scapellato, A. Qualitative Properties of Solutions of Second-Order Neutral Differential Equations. Symmetry 2020, 12, 1520. [CrossRef]

17. Berezansky, L.; Braverman, E. Oscillation of a linear delay impulsive differential equations. Commun. Appl. Nonlinear Anal. 1996, 3, 61-77.

18. Diblik, J.; Svoboda, Z.; Smarda, Z. Retract principle for neutral functional differential equation. Nonlinear Anal. Theory Methods Appl. 2009, 71, 1393-1400. [CrossRef]

19. Santra, S.S.; Alotaibi, H.; Bazighifan, O. On the qualitative behavior of the solutions to second-order neutral delay differential equations. J. Ineq. Appl. 2020, 2020, 256. [CrossRef]

20. Diblik, J. Positive solutions of nonlinear delayed differential equations with impulses. Appl. Math. Lett. 2017, 72, 16-22. [CrossRef]

21. Luo, Z.; Jing, Z. Periodic boundary value problem for first-order impulsive functional differential equations. Comput. Math. Appl. 2008, 55, 2094-2107. [CrossRef]

22. Yu, J.; Yan, J. Positive solutions and asymptotic behavior of delay differential equations with nonlinear impulses. J. Math. Anal. Appl. 1997, 207, 388-396. 
23. Tripathy, A.K. Oscillation criteria for a class of first order neutral impulsive differential-difference equations. J. Appl. Anal. Comput. 2014, 4, 89-101.

24. Berezansky, L.; Domoshnitsky, A.; Koplatadze, R. Oscillation, Nonoscillation, Stability and Asymptotic Properties for Second and Higher Order Functional Differential Equations; Chapman Hall/CRC Press: Boca Raton, FL, USA, 2020.

25. Agarwal, R.P.; Bohner, M.; Li, T.; Zhang, C. A new approach in the study of oscillatory behavior of even-order neutral delay differential equations. Appl. Math. Comput. 2013, 225, 787-794. [CrossRef]

26. Agarwal, R.P.; Zhang, C.; Li, T. Some remarks on oscillation of second order neutral differential equations. Appl. Math. Comput. 2016, 274, 178-181. [CrossRef]

27. Bohner, M.; Hassan, T.S.; Li, T. Fite-Hille-Wintner-type oscillation criteria for second-order half-linear dynamic equations with deviating arguments. Indag. Math. (N. S.) 2018, 29, 548-560. [CrossRef]

28. Bohner, M.; Li, T. Oscillation of second-order $p$-Laplace dynamic equations with a nonpositive neutral coefficient. Appl. Math. Lett. 2014, 37, 72-76. [CrossRef]

29. Chatzarakis, G.E.; Grace, S.R.; Jadlovská, I.; Li, T.; Tunç, E. Oscillation criteria for third-order Emden-Fowler differential equations with unbounded neutral coefficients. Complexity 2019, 2019, 1-7. [CrossRef]

30. Chiu, K.-S.; Li, T. Oscillatory and periodic solutions of differential equations with piecewise constant generalized mixed arguments. Math. Nachr. 2019, 292, 2153-2164. [CrossRef]

31. Džurina, J.; Grace, S.R.; Jadlovská, I.; Li, T. Oscillation criteria for second-order Emden-Fowler delay differential equations with a sublinear neutral term. Math. Nachr. 2020, 293, 910-922. [CrossRef]

32. Li, T.; Rogovchenko, Y.V. Oscillation of second-order neutral differential equations. Math. Nachr. 2015, 288, 1150-1162. [CrossRef]

33. Li, T.; Rogovchenko, Y.V. Oscillation criteria for even-order neutral differential equations. Appl. Math. Lett. 2016, 61, 35-41. [CrossRef]

34. Li, T.; Rogovchenko, Y.V. Oscillation criteria for second-order superlinear Emden-Fowler neutral differential equations. Monatsh. Math. 2017, 184, 489-500. [CrossRef]

35. Li, T.; Rogovchenko, Y.V. On the asymptotic behavior of solutions to a class of third-order nonlinear neutral differential equations. Appl. Math. Lett. 2020, 105, 1-7. [CrossRef]

36. Tripathy, A.K.; Santra, S.S. Necessary and sufficient conditions for oscillations to a second-order neutral differential equations with impulses. Kragujev. J. Math. 2020, 47, 81-93. 